\title{
Research on visualized search and rescue robot
}

\author{
Fengzhi Dai $^{1}$, Qijia Kang ${ }^{1}$, Yuanli Yue ${ }^{1}$, Pengfei Xie ${ }^{1}$ \\ ${ }^{1}$ Tianjin University of Science and Technology, \\ 1038 Dagunanlu Road, Hexi District, Tianjin, 300222, China \\ E-mail:daifz@tust.edu.cn \\ www.tust.edu.cn
}

\begin{abstract}
Faced to the weakness of the low efficiency and limitation of the recent search and rescue robot, this paper presents a visualized search and rescue robot based on the Arduino platform. The infrared detector is used to detect life, and the camera can transmit the information back to the mobile phone or computer terminal in real-time. The track wheel improves the capability of shuttle and over obstacle. It can partly replace humans to finish the task under the dangerous environment and search-and-rescue person in the confined space.
\end{abstract}

Keywords: visualized, infrared detector, track, search and rescue

\section{Introduction}

Since 1980s, the concept of search and rescue robot has been presented. With the rapid development of science and technology, the application of the search and rescue robots become wider ${ }^{1}$. By its superior mobility and well adapt ability in various environments, the search and rescue robot has already been used on different fields, for example, the earthquake rescue robots. It is a robot that are used to search for survivors under the rubble after the earthquake, which is equipped with a color camera, thermography and communication system ${ }^{2}$.

The robot can replace the rescue workers into the dangerous environment, then implement the rescue work and transport the necessary supplies for the trapped persons ${ }^{3}$.

The search and rescue robot is different from the household robot or industrial robot. Its working environment is uncertain and dangerous. Search and rescue robots generally have the following advantages ${ }^{4,5}$ :

- Walking through the ruins to detect signs of life freely with small size and flexible operator.

- Having strong adaptability in various environment, which can arrive at the high temperature and high pressure place where the rescue workers can not arrive in.

- Being equipped with functional sensors and detection equipment, such as the infrared detector and camera searching for survivors. Using lithiumion batteries with low power consumption and long battery working time ${ }^{6}$.

\section{The structural design}

In the paper, a new visualized rescue robot is developed that is adapt to the complex environment and has more powerful rescue capability.

First, the search and rescue robot enters the rescue scene. Second, it gathers the information by sensors. And finally, it uses the camera to transmit images to the mobile phone or computer terminal real-time by wireless technology.

Also, the robot should have the following functions: excellent overstepping and feedback capabilities in realtime.

The structural design of the search and rescue robots is shown in Fig.1. 

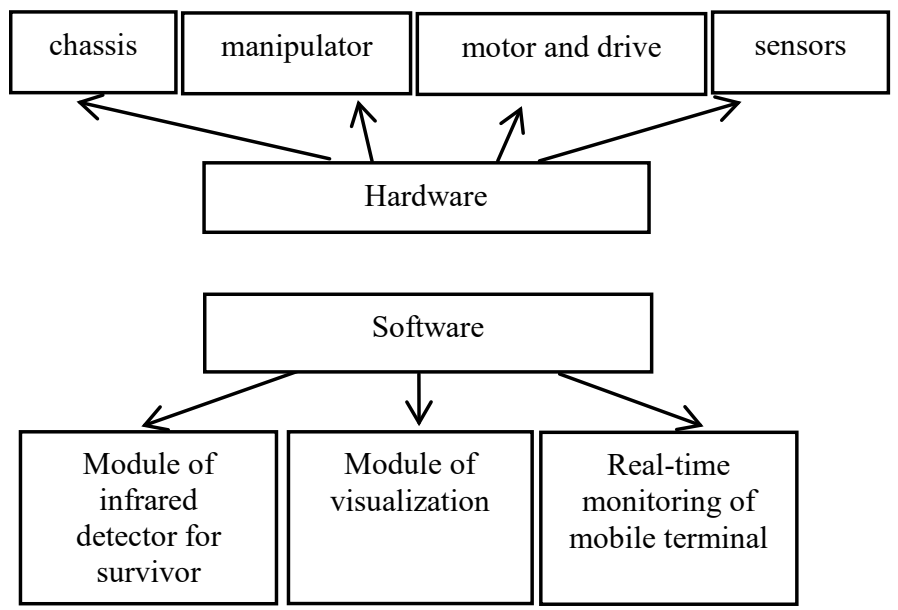

Fig.1 The structural design of search and rescue robots

\subsection{Manipulator}

The search and rescue robot has a six degree of freedom mechanical arm (manipulator). When a small obstacle is in front of the robot, the robot can easily catch and throw it by the arm. If the obstacle is large, the arm helps the robot to climb over it.

The manipulator is made up of the upper arm, lower arm and rotary frame. The upper arm and lower arm are mounted on the rotating frame by two large torque servo to drive ${ }^{7}$, which has enough strength to carry goods.

For the manipulator, the rotating angle of each rotary frame can reach to $180^{\circ}$, and the gyration radius is $355 \mathrm{~mm}$, the maximum height is $460 \mathrm{~mm}$ (the gripper closed), the maximum value of the opening in front of the gripper is $55 \mathrm{~mm}$ and the widest range of the clipped goods is $98 \mathrm{~mm}$. The manipulator is shown in the Fig. 2 .

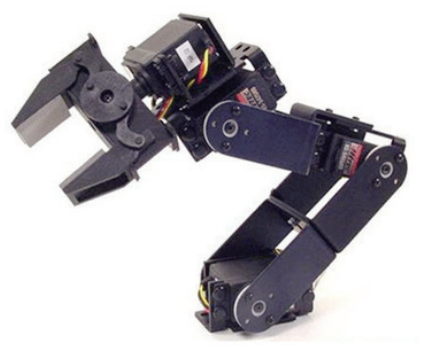

Fig.2. Rotating mechanical arm

\subsection{Chassis structure}

In the mechanical design process, the main part is the chassis, which determines maneuvering capability and it is important for overstepping obstacles.

Fig. 3 is the chassis of the robot. In the front of the car tracks, there is an oblique angle (as shown in Fig.3). This structure can greatly enhance the ability to climb over obstacles.

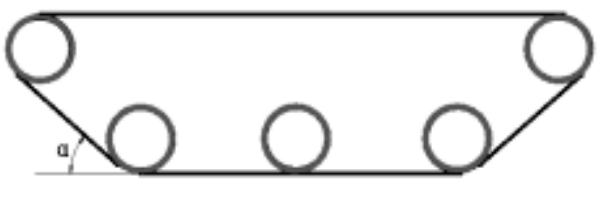

Fig.3. Crawler design of the chassis

The rhombus frame structure is accepted and the material selected guarantees the frame's strength as well as lower weight. The crawler mechanical structure is suitable to improve the ability of climbing and has good ability to climb over obstacles.

\subsection{The circuit control}

Circuit control includes the main control chip, driving motor, sensing scheme and remote signal transmission module. The sensing scheme includes a variety of sensing modules. For example, rotating machine arm module, visual operation module and the human infrared sensor module for detecting life characteristics.

\subsubsection{Main control chip}

The control circuit chooses the ATmega128-AU as the main chip, which is used to control and deal with a variety of information sampled from sensors. Its voltage is $3.3-5 \mathrm{~V}$ and it has $16 \mathrm{M}$ high speed crystal, which can provide a high clock frequency. The main control chip have a high processing speed, so it can process the data from sensors in real time.

ATmega128 is a CMOS 8 bit microcontroller that is based on the AVR with low power and high performance. The structure of the AVR is the Harvard, with independent data and program bus. ATmega128 contains $4 \mathrm{~K}$ bytes EEPROM, which can meet the needs of the program. ATmega128 is shown in Fig.4.

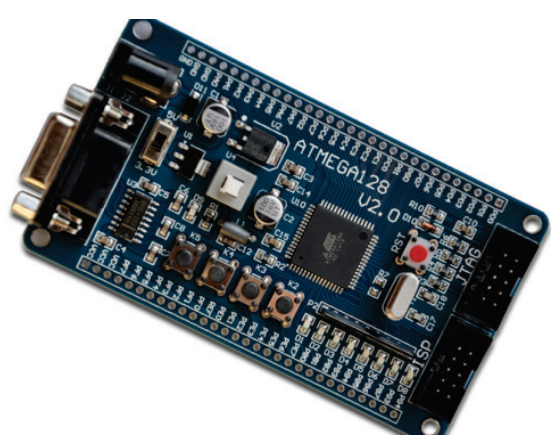

Fig.4. Main control chip ATmega128 


\subsubsection{Motor selection}

Large torque motor can provide enough power in the progress of moving. Therefore, the robot uses $37 \mathrm{~GB}-$ 500 permanent-magnet direct current speed reduction motor, whose rated voltage is $1.5-36 \mathrm{~V}$, rated speed is 5$3000(\mathrm{rpm})$ and rated torque is $0.02-30(\mathrm{NM})$.

\section{Introduction of functional modules}

With a variety of auxiliary functional modules, the search and rescue robot can get the location information and life characteristics of the trapped personnel. The robot is shown in Fig.5.

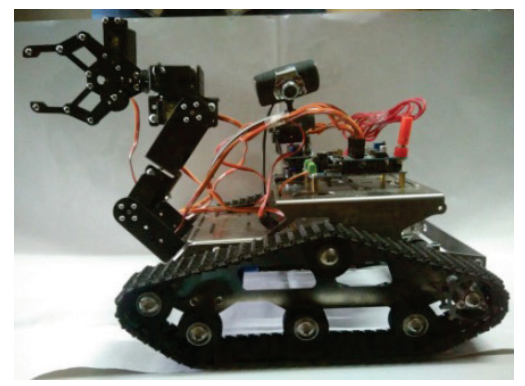

Fig.5. The overall structure figure

\subsection{Infrared detector for searching survivors}

The robot can search and rescue the trapped person in dangerous areas or in ruins. Therefore, the infrared detector for searching survivors is needed.

As we know, all the objects will transmit the infrared spectrum (only if its absolute temperature exceeds $273^{\circ} \mathrm{C}$ ). But they have different wavelengths in the infrared spectrum. According to this natural phenomenon, it can be applied to search and rescue the trapped person.

Normally, for the human beings, the body temperature is $36 \sim 37^{\circ} \mathrm{C}$ and the wavelength of infrared spectrum transmitted is $9 \sim 10 \mu \mathrm{m}$. Thus, the pyroelectric infrared detector is used, and the sensors of the infrared detector is shown in Fig. 6 .

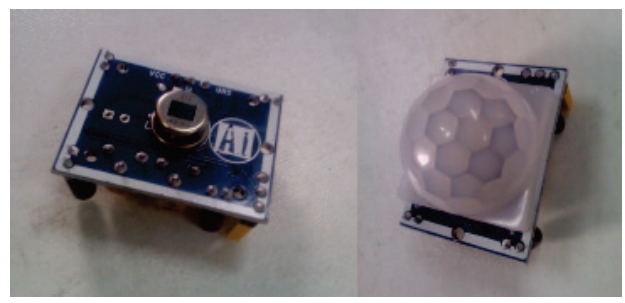

Fig.6. The infrared detector

\subsection{The function of visualization}

Vision is important to the robot. Visualization is the primary function to get the environment information for rescuer in real-time.

The situation in dangerous areas or in ruins may be dark or has poor light. If the rescuer uses the intense light to search, it may cause the secondary damage to the eyes of the affected people. In order to avoid this problem, the infrared camera is selected. The camera should also have the functions of wireless transmission and video recording, which can provide valuable data for the disaster relief.

Two geared motors are used to control the camera to rotate freely horizontally and vertically.

The camera has the following features:

- The module of wireless transmission optimizes H. 264 lossless compression arithmetic. This method can decrease the amount of transmission data and time effectively, and also ensure the high image quality.

- With the infrared light, the camera automatically switches between the modes of day and night by the IR-CUT. By this way, it can guarantee the accuracy and clarity in the image transmission when the disaster scene is in poor light ${ }^{8,9}$.

- The camera has the TF card slot, by which the video and image information can be saved in the rescue and relief operations. Therefore, it is convenient to optimize the rescue path in the progress of the follow-up succor based on the saved videos.

- The visual angle is 360 degrees and the camera can rotate 80 degrees to ensure video capture in narrow space.

\section{Some important functions}

\subsection{Obstacle overstepping}

Capability of overstepping is an important measure on the robot. When the robot encounters obstacle, it can clear obstacles by the manipulator. If the obstacle is large, the robot should cross or overstep the obstacle.

\subsection{Collecting parameters in spot environment}

The robot needs to arrive at the accident scene and implement the search and rescue mission, which requires the robot to gather information accurately and efficiently. The main functions are as follows: capturing video, searching for the survivors by the infrared detector and transmitting the information in real-time. 


\subsection{Feedback capability}

Visualization is a major function to the search and rescue robots, which can send information of location back to the outside world. Therefore, relief workers can get environment information and physical condition of the trapped personnel in real-time. So that the relief workers can use these provided information for rescue that the robot cannot do.

\section{Testing and conclusion}

By the test of the search and rescue robot, the following data is obtained: The maximum height of the barrier that the robot can climb cross is $120 \mathrm{~mm}$, the maximum angle of climbing is 38 degrees, the maximum range of detection is a circle of radius $1 \mathrm{~m}$, the maximum distance of transmission is $22 \mathrm{~m}$. The above data shows that the robot can basically meet the needs of rescue 10 .

In the paper, a kind of visualized rescue robot is designed. The design of walking manner is based on the crawler structure with an oblique angle, and cooperates with the manipulator, which improves the ability of climbing.

And by using the camera and the technology of wireless transmission, the image and video can be transmitted to the control terminal in real-time. At the same time, with the sensors of infrared detector for searching the wounded personnel, the rescue robot can detect the affected person on a wide range.

The test includes the ability of climbing, the accuracy of infrared detector and the relationship between wireless transmission distance and the obstacle. Finally, the test result shows that the visualized search and rescue robot has a good ability to overcome obstacles and can basically meet the needs of rescue.

\section{Acknowledgements}

The research is partly supported by the Research Fund for the Doctoral Program of Higher Education of China (20131208110005). It is also supported by the Science $\&$ Technology Fund Planning Project of Tianjin Higher Schools (20140710-1400020005).

\section{References}

1. Guohua $\mathrm{Xu}, \mathrm{MinTan}$, The development and tendency of mobile robots, Robot Technique and Application, (3) (2001) 7-14.

2. YouHe, Guohong Wang, Dajin Lu, Multi-sensor information fusion (Continued). Infrared and Laser Engineering, 28 (2)(1999) 10-15.

3. Y Huo, L Zhang, A path planning algorithm for mobile robot, Techniques of automation and applications, 22(5) (2003) 8-10.

4. Lei $\mathrm{Li}$, Tao Ye, The research and future research of mobile robot technology, Robot, 24(5) (2002) 475-480.

5. Xiaodong Zhuang, Qingchun Meng, The robot path search method based on fuzzy concept in dynamic environment. Robot, 23 (5) (2001) 397-399.

6. JunWang, Xinhan Huang, Obstacle avoidance control of two wheeled mobile robot based on neural network learning, Robot, 18 (5) (1996) 292-297.

7. Shiyong Li, The theory of fuzzy control and intelligent control (Harbin, Harbin Institute of Technology Press, 1999)

8. Qingzhong Li, A path planning method for mobile robot based on genetic algorithm, Pattern Recognition and Artificial Intelligence, 15 (2) (2002) 161-165.

9. Z Wang, Geguo, The present situation and prospect of mobile robot navigation technology. Robot, 25 (5) (2003) 470-474.

10. Leize Jin, Zhenjun Du, Kaijia, The research on simulation of path planning for mobile robot based on potential field method, Computer Engineering and Applications, 43 (24) (2007) 226-228. 